SOZIALE ARBEIT AKTUELL

\section{Maren Zeller}

Die sozialpädagogische Verantwortung der Schule

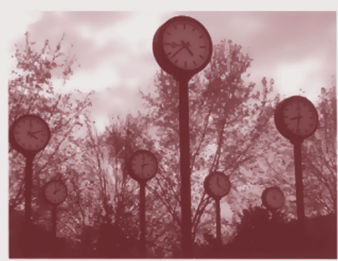

㤙

$$
\begin{array}{r}
\text { Kooperation von Ganztagsschule } \\
\text { und Jugendhilfe }
\end{array}
$$

\section{MAREN ZELLER (HG.)}

Die sozialpädagogische Verant wortung der Schule. Kooperation von Ganztagsschule und Jugendhilfe.

Baltmannsweiler: Schneider Verlag, 155 Seiten, IBSBN 3-8340-0158-9, EUR 14
Der Sammelband fokussiert vornehmlich die Frage, vor welche Herausforderungen die Kooperation von Schule und Jugendhilfe im Zuge der verstärkten Einführung von Ganztagsschulen gestellt wird. Neben dem aktuellen Diskussionsstand, stellt es auch empirische Ergebnisse vor und zeigt so die jeweiligen Chancen und Begrenzungen der Kooperationsmöglichkeiten auf. Zugleich dienen die Beiträge auch als pragmatische Anregungen für die Praxen der Schule als auch Jugendhilfe, ohne die jeweiligen professionsbezogenen Ansprüche nivellieren zu wollen.

Grundkanon aller Beiträge ist, dass sich aus den gesellschaftlichen Anforderungen an Kinder und Jugendliche beide pädagogischen Sphären mit ihren jeweiligen Kompetenzen ergänzen können und müssen. Der Buchtitel verweist auf eine Erweiterung der schulischen Ziele um familien- und sozialpolitische Aspekte, die, so die Herausgeberin, „zunächst der sozialpädagogischen Perspektive, die schon immer den Ausgleich sozialer Ungleichheit thematisiert, immanent" seien. Dennoch zeigen die Beiträge, dass die Vision einer sozialpädagogischen Ganztagsschule nicht das ideale Lösungsmodell ist, sondern vielmehr die Verzahnungen auf die jeweiligen Kompetenzen der einzelnen Jugendhilfebereiche abgestimmt werden müssen. Der Band ist in zwei diskursive Schwerpunkte gegliedert: Erstens bündeln und reflektieren drei Beiträge übergreifend aktuelle Diskurse zur Kooperation.

Wolfgang Mack zeichnet die Ausdifferenzierung der Jugendhilfe mit ihren Aufgaben als deren Chance nach. Dabei verortet er das Kooperationspotential in den Maximen der Jugendhilfe. Maren Zeller analysiert die Vielzahl an programmatischen Stellungnahmen in der Jugendhilfe und arbeitet aus ihren konzeptionellen Bezugnahmen einen ganzheitlichen Bildungsbegriff als Kanon heraus. Als problematisch zeigt sich dabei, dass der Begriff der ganzheitlichen Bildung jeweils höchst unterschiedlich gefüllt wird.

Carola Flad und Eberhard Bolay stellen die baden-württembergische Entwicklung in der Kooperationspraxis von Jugendhilfe und Schule vor und fächern anschließenden Thesen auf, inwiefern Ganztagsschulen mit sämtlichen Angeboten der Jugendhilfeanbieter zu verzahnen sind. Zweitens gehen Mechthild Eickhoff, Burkhard Müller, Nadja Skale und Andreas Wiehe auf einzelne Jugendhilfeaufgaben - Schul-

jugendarbeit, Jugendarbeit, Jugendkulturarbeit und schulische Erziehungshilfen - mit eigenem empirischem Material ein und zeigen daran fachliche Herausforderungen, aber auch Schnittstellen.

Markant im vorliegenden Band sind die unterschiedlichen Horizonte, die die jeweiligen Beiträge eröffnen: In der Gesamtschau stellen die Beiträge das komplexe Wechselverhältnis zwischen Rahmenbedingungen und Kooperationspraxen auch für Einsteiger aus der Praxis oder für Studierende verständlich dar. Zugleich reflektieren und relativieren sie den einengenden Bildungsdiskurs der Jugendhilfe, indem sie auch die anderen Qualitäten der Arbeitsfelder, Erziehung und Hilfe, argumentativ stärken. Dabei sind die Beiträge in ihrer Unterschiedlichkeit gerade deshalb anregend, da sie aktuelle Diskussionsstränge weiter vertiefen. Somit ist es auch für Pädagoginnen und Pädagogen aus den schulischen, als auch aus den Jugendhilfebereichen ein anregender und aktueller Blick in den pädagogisch vielleicht (noch) fremden Garten der Anderen.

MARC SCHULZ

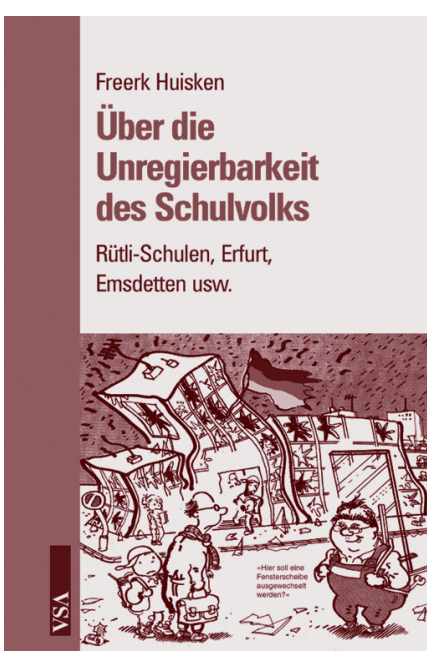

FREERK HUISKEN

Über die Unregierbarkeit des Schulvolks - Rütli-Schulen, Erfurt, Emsdetten usw.

Hamburg: VSA, 2007, 173 Seiten, ISBN 978-3-89965-210-9, EUR 12,80
Freerk Huisken, bis 2006 Pädagogik-Professor an der Universität Bremen, hat seit den 90er Jahren neben seiner fundamentalen Schulkritik („Erziehung im Kapitalismus“, Hamburg 1998) publizistisch vor allem eine Sache aufs Korn genommen: die Konstruktion eines Jugendproblems, das durch "Gewaltbereitschaft" gekennzeichnet sei, rassistische, nationalistische oder (selbst-)destruktive Verlaufsformen kenne und nach speziellen sozial-pädagogischen Problemlösungsstrategien verlange. Er hat dies sowohl im Zusammenhang der „Rechtsextremismuskampagne“, des „Aufstands der Anständigen“ der rotgrünen Ära, aufgegriffen („Nichts als Nationalismus", Bd. 1 und 2, Hamburg 2001) als auch im Blick auf die neuen Diskussionen über unpolitische Jugendgewalt und spektakuläre „Einzelfälle“ wie die Schulattentate in Erfurt oder Emsdetten. Diese Auseinandersetzung hat Huisken jetzt mit einem Sammelband fortgeschrieben, der vier aktuelle Aufsätze enthält. Sie basieren auf Vorträgen, die der Autor in den letzten Jahren gehalten hat, und sind um einen ausführlichen Debattenteil ergänzt. Roter Faden ist das Ordnungsproblem, das die Schulpolitik seit einiger Zeit in verschärfter Form bei Schülern aus „bildungsfernen“, „sozial schwa- 